\title{
Seleção de clones de braquiária para resistência à lagarta-do-cartucho
}

\author{
Alexander Machado Auad(1), Fausto Souza Sobrinho(1), Simone Martins Mendes(2), \\ Antônio Marcos Oliveira Toledo(1), Thiago de Souza Lucindo(1) e Flavio Rodrigo Gantolfi Benites ${ }^{(1)}$
}

(1)Embrapa Gado de Leite, Rua Eugênio do Nascimento, no 610, Dom Bosco, CEP 36038-330 Juiz de Fora, MG, Brasil. E-mail:
alexander.auad@embrapa.br,fausto.souza@embrapa.br,antoniomarcosbio@live.com,thiiiagosouza64@gmail.com,flavio.benites@embrapa.br
(2)Embrapa Milho e Sorgo, Rodovia MG-424, Km 45, CEP 35701-970 Sete Lagoas, MG, Brasil. E-mail: simone.mendes@embrapa.br

Resumo - O objetivo deste trabalho foi avaliar e selecionar clones de braquiária (Urochloa ruziziensis) resistentes à lagarta-do-cartucho, Spodoptera frugiperda (Lepidoptera: Noctuidae), para uso em sistema de integração lavoura-pecuária-floresta. Foram avaliados 147 clones de $U$. ruziziensis em delineamento inteiramente casualizado, com três repetições. Como testemunha e padrão de suscetibilidade, foi utilizada uma dieta artificial adequada ao desenvolvimento do inseto. Folhas dos diferentes clones de $U$. ruziziensis foram coletadas e oferecidas como alimento aos insetos, mantidos individualizados em unidades de criação. Foram avaliadas a sobrevivência, a duração e a biomassa de lagartas e pupas. Observaram-se diferenças significativas entre os clones para todas as características avaliadas, o que evidencia a influência acentuada dos clones de $U$. ruziziensis no desenvolvimento de $S$. frugiperda. Foram identificados 20 clones nos quais não houve desenvolvimento da lagarta e 55 clones nos quais houve sobrevivência média das lagartas de 33\%. Para os demais clones, que se mostraram melhores hospedeiros, o desenvolvimento do inseto foi afetado pela maior duração do estágio larval e pela redução da biomassa da pupa. Os resultados obtidos são indicativos do potencial de utilização de clones de Urochloa ruziziensis para redução de populações de Spodoptera frugiperda em sistema de integração lavoura-pecuária-floresta.

Termos para indexação: Spodoptera frugiperda, Urochloa ruziziensis, integração lavoura-pecuária-floresta, resistência de plantas.

\section{Selection of signal grass clones for resistance to fall armyworm}

\begin{abstract}
The objective of this work was to evaluate and select signal grass (Urochloa ruziziensis) clones resistant to fall armyworm, Spodoptera frugiperda (Lepidoptera: Noctuidae), for use in a crop-livestock-forest integration system. One hundred and forty-seven clones of $U$. ruziziensis were evaluated in a completely randomized design with three replicates. An artificial diet, adequate for insect development, was used as a control and susceptibility standard. Leaves of different $U$. ruziziensis clones were collected and offered as food to the insects, kept individually in rearing units. Larval survival, duration, and biomass of larvae and pupa were evaluated. Significant differences were observed between clones for all evaluated traits, showing the marked effect of $U$. ruziziensis clones on the development of $S$. frugiperda. Twenty clones were identified in which there was no development of larvae and 55 clones in which there was an average larval survival of $33 \%$. For the other clones, which were better hosts, insect development was affected by the longer larval stage and by the reduction in pupa biomass. The obtained results show the potential of using Urochloa ruziziensis clones for the reduction of Spodoptera frugiperda populations in a crop-livestock-forest integration system.
\end{abstract}

Index terms: Spodoptera frugiperda, Urochloa ruziziensis, crop-livestock-forest integration, plant resistance.

\section{Introdução}

O sistema de integração lavoura-pecuária-floresta (ILPF) é uma das principais estratégias utilizadas em sistemas agropecuários dos trópicos (Balbino et al., 2012). Esse sistema tem mostrado viabilidade econômica na utilização de milho e sorgo em consórcio com forrageiras do gênero Urochloa (Gontijo Neto et al., 2014), com benefícios ao produtor e ao meio ambiente (Alvarenga et al., 2010). Porém, o uso desse sistema de consorciação também contribui para que a lagarta-do-cartucho [Spodoptera frugiperda (J.E. Smith, 1797) (Lepidoptera: Noctuidae)], espécie polífaga que ataca diversas culturas de valor econômico, como milho e forrageiras (Lopes et al., 2008; Sá et al., 2009; Casmuz et al., 2010; Campos et al., 2011; 
Boregas et al., 2013), tenha hospedeiros durante todo o ano, para o seu desenvolvimento (Waquil et al., 2008).

Spodoptera frugiperda é uma das pragas mais nocivas nas regiões tropicais das Américas, uma vez que causa perdas de 15 a $37 \%$ na produção (Figueiredo et al., 2006), com prejuízos que podem chegar a 500 milhões de dólares anuais (Waquil et al., 2002). Nesse cenário, a seleção de plantas resistentes a esse inseto contribui para a definição e a implementação do programa de manejo integrado a ser adotado no sistema de ILPF.

Sá et al. (2009) e Boregas et al. (2013) avaliaram a biologia de $S$. frugiperda alimentada com Urochloa sp. e observaram que este gênero é um bom hospedeiro para o inseto. Em sistemas de ILPF, Urochloa sp. é indicada para o consórcio com milho e árvores; assim, para o melhoramento de plantas, um dos desafios é selecionar clones de Urochloa ruziziensis que minimizem o potencial biótico desse inseto-praga, de forma a não potencializar o problema na cultura consorciada na qual o inseto é bem adaptado.

O objetivo deste trabalho foi avaliar e selecionar clones de braquiária (Urochloa ruziziensis) resistentes à lagarta-do-cartucho (Spodoptera frugiperda), para uso em sistema de integração lavoura-pecuária-floresta.

\section{Material e Métodos}

Foram avaliados 147 clones de Urochloa ruziziensis (Syn. Brachiaria ruziziensis R.Germ. \& C.M.Evrard), oriundos do programa de melhoramento desta espécie forrageira da Embrapa Gado de Leite, localizada em Juiz de Fora, MG. A produção das mudas foi iniciada em outubro de 2013, por meio da clonagem de estacas maduras das plantas em casa de vegetação. Após 45 dias, realizou-se o transplantio das mudas para vasos de 3,0 L, contendo uma mistura de terra, areia e esterco, na proporção de 1:1:1. Na primeira quinzena de fevereiro de 2014, foi realizado um corte para uniformização das plantas. No início de abril de 2014, quando as plantas estavam com 40 dias de crescimento, o experimento foi iniciado na Embrapa Gado de Leite.

Foram utilizados ovos de $S$. frugiperda, provenientes de criação da Embrapa Milho e Sorgo, em Sete Lagoas, MG. No Laboratório de Entomologia da Embrapa Gado de Leite, cada lagarta, com até 24 horas de idade, foi mantida individualizada em unidades de criação com 3,6 cm de diâmetro x 3,7 cm de altura, fechadas com tecido voil, preso por elástico. Folhas de todos os clones testados foram coletadas e fornecidas como alimento aos insetos. Para manutenção da turgescência, as folhas foram fixadas em $1,0 \mathrm{~cm}$ de ágar. $\mathrm{O}$ alimento oferecido a $S$. frugiperda foi trocado a cada 48 horas, para garantir condições adequadas de alimentação. Como testemunha e padrão de suscetibilidade, foi utilizada uma dieta artificial à base de feijão, levedo de cerveja, germe de trigo, ácido ascórbico, nipagin, ácido sórbico, ágar, formaldeído e inibidor de fungo. Essa dieta foi ofertada às lagartas em cubos de 2,0x2,0 cm e foi posicionada nas unidades de criação citadas anteriormente.

Utilizou-se o delineamento inteiramente casualizado, com três repetições. Durante todo o período experimental, os insetos foram mantidos em câmara climatizada tipo fitotron, com condições controladas: $25 \pm 2^{\circ} \mathrm{C}, 70 \pm 10 \%$ de umidade relativa e fotofase de 14 horas.

Diariamente, avaliou-se a sobrevivência das lagartas, e, quando o experimento completou 15 dias, foi aferida a biomassa das sobreviventes com auxílio de balança de precisão. A partir de então, vistoriou-se diariamente os insetos até a data da formação das pupas, que foram pesadas após 48 horas.

Os dados de sobrevivência (\%), duração (dias) e biomassa de lagartas (mg) e pupas ( $\mathrm{mg}$ ) foram submetidos à análise de variância (Anova), e as médias foram comparadas pelo teste de Scott-Knott, a $5 \%$ de probabilidade. Os clones de $U$. ruziziensis foram agrupados de acordo com os resultados do teste de média. Para estimar a adaptação de $S$. frugiperda nos diferentes hospedeiros, foi utilizado o índice de adaptação (IA) proposto por Boregas et al. (2013), em que $\mathrm{IA}=($ sobrevivência larval $\times$ biomassa de pupas $) /$ duração larval; esse índice sumariza as variáveis supracitadas, para os clones com sobrevivência acima de $33 \%$. A correlação de Spearman entre a duração e a biomassa de lagartas e pupas do inseto-praga foi estimada por meio do programa BioEstat (Ayres et al., 2003).

\section{Resultados e Discussão}

A sobrevivência de $S$. frugiperda, na fase de lagarta, foi afetada pelos diferentes clones de $U$. ruziziensis $(\mathrm{F}=1,143 ; \mathrm{p}=0,005)$, o que indica que nem todos foram hospedeiros adequados para o inseto-praga 
(Figura 1). Esses resultados confirmam a grande variabilidade genética existente dentro dessa espécie quanto a características de importância para as forrageiras (Souza Sobrinho \& Auad, 2013). Essa variabilidade entre os clones de $U$.ruziziensis pode ser explorada pelo melhoramento genético, que permite a identificação e a seleção de plantas não favoráveis ao desenvolvimento de S. frugiperda. Além disso, como essa espécie forrageira é amplamente cultivada em sistemas integrados, consorciada principalmente com o milho (Zea mays L.), a obtenção de clones resistentes às lagartas de $S$. frugiperda é de grande importância, para evitar que os insetos reduzam a produtividade de forragem verde utilizada como alimento para o gado na entressafra ou mesmo a massa de palhada para o plantio posterior. $\mathrm{O}$ emprego de cultivares forrageiras resistentes também evitará a multiplicação das pragas na entressafra, o que poderia acarretar populações maiores do inseto na cultura principal.

As médias de sobrevivência do inseto nos diferentes clones variaram de 0 a $100 \%$, e foram usadas para a formação de três grupos distintos. O grupo de materiais com menor sobrevivência do inseto-praga, de 0 a $33 \%$,

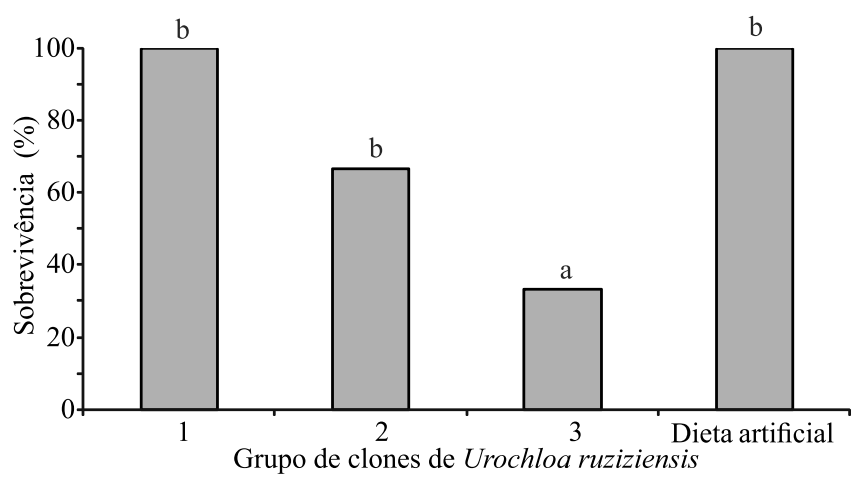

Grupo 1: 4, 11, 29, 35, 42, 57, 61, 62, 71, 76, 102, 112, 116, 117, 118, 128, 133, 144 Grupo 2: 3, 6, 13,14, 15, 18, 19, 21, 25, 28, 34, 39, 45, 49, 54, 59, 60, 66, 70, 73, 74 $78,79,83,84,85,86,87,88,91,93,96,107,109,115,119,120,122,124$ $125,126,130,131,134,136,138,139,140,141,142,143,145,148,149$ Grupo 3:1, 2, 5, 7, 8, 9, 12, 16, 17, 20, 23, 24, 27, 37, 38, 40, 43, 44, 46, 47, 50, 51, $52,53,56,58,64,67,68,69,72,77,80,81,82,89,90,92,94,95,97,99$, $101,103,104,106,108,110,114,121,129,132,137,146,147,10,22,26$, $30,31,32,33,36,41,48,55,63,98,100,105,113,123,135,150$

Figura 1. Sobrevivência média de lagartas de Spodoptera frugiperda alimentadas com diferentes clones de Urochloa ruziziensis. Médias seguidas de letras distintas diferem pelo teste de Scott-Knott, a 5\% de probabilidade, e formam os diferentes grupos de clones. No grupo 1, encontram-se todos os materiais com $100 \%$ de sobrevivência; no grupo 2, com $66 \%$ de sobrevivência; e, no grupo 3 , com 0 a $33 \%$ de sobrevivência. correspondeu a 51\% dos clones de $U$. ruziziensis. Os demais clones, juntamente com a dieta artificial (padrão de suscetibilidade), proporcionaram sobrevivência média acima de $66,7 \%$ (Figura 1). Sá et al. (2009) já haviam relatado a adequação dessa forrageira para lagartas de S. frugiperda $(98 \%$ de sobrevivência).

Essa amplitude de sobrevivência, de acordo com os hospedeiros ofertados, reflete as alterações nos processos fisiológicos associados às plantas hospedeiras e ao biótipo do inseto avaliado. A redução da sobrevivência é esperada em hospedeiros de baixa qualidade para o inseto-praga ou com presença de fator antinutricional, como constatado no presente trabalho, em $51 \%$ dos clones testados.

Foram identificados 20 clones nos quais não houve desenvolvimento das lagartas e 55 clones nos quais houve sobrevivência média das lagartas, abaixo de 33\% (grupo 3, Figura 1); esses materiais serão indicados para o programa de melhoramento de U. ruziziensis. Já nos materiais que proporcionaram condições intermediárias ( $66 \%$ de sobrevivência) e favoráveis para o desenvolvimento dos insetos (100\% de sobrevivência) (grupos 1 e 2, Figura 1), a duração da fase da lagarta foi alterada em função dos clones ofertados $(\mathrm{F}=2,748 ; \mathrm{p}=0,0001)$, com variação de 17,5 a 24,7 dias para a dieta artificial e para os clones mais longínquos, respectivamente (Figura 2). Pencoe \& Martin (1981) verificaram durações de 13,1 e 18,5 dias, respectivamente, quando foram ofertadas, para $S$. frugiperda, uma dieta artificial e a forrageira Cyperus esculentus.

A alteração no valor nutricional do alimento ofertado reflete-se diretamente na redução do período de desenvolvimento das lagartas. Conforme Busato et al. (2005), um dos parâmetros bromatológicos que pode explicar essa qualidade é a proteína. Os autores observaram desempenho inferior das lagartas (maior duração e menor biomassa) quando alimentadas com milho ofertado na forma de folhas, em comparação ao arroz (Oryza sativa L.), com maior teor proteico. Essa inferência também foi feita por Pashley et al. (1995), que estudaram o efeito do milho e da grama-estrela (Cynodon nlemfuensis Vanderyst) no desenvolvimento de $S$. frugiperda. Esses resultados são indicativos de que um menor período de desenvolvimento possibilita ao inseto completar um maior número de gerações por período, com menor exposição a fatores bióticos 
e abióticos de mortalidade; portanto, para o programa de melhoramento, devem-se considerar os clones que proporcionaram maior duração da fase larval (Figura 2).

Nos 72 clones pertencentes aos grupos 1 e 2 (Figura 1), a biomassa das lagartas aos 15 dias após a eclosão não se diferenciou em função dos clones $(\mathrm{F}=1,239$; $\mathrm{p}=0,0737)$. No entanto, os clones proporcionaram biomassas diferentes na fase subsequente, de pupa $(\mathrm{F}=2,030 ; \mathrm{p}=0,0005)$, cujas médias foram separadas em dois grupos (Figura 3). Já a biomassa das lagartas alimentadas com dieta artificial não diferiu da daquelas alimentadas a partir dos clones de $U$. ruziziensis; porém, a dieta artificial proporcionou maiores biomassas às pupas. Sá et al. (2009) registraram menor biomassa de lagartas e de pupas, em torno de 410 e $190 \mathrm{mg}$, respectivamente, quando alimentadas com essa forrageira, em comparação ao sorgo, ao milho e à dieta artificial; contudo, apesar de a braquiária não ser o alimento preferido, essa praga ainda conseguiu se desenvolver. Lopes et al. (2008) relataram que essa espécie-praga, quando mantida em folhas de mandioca (Manihot esculenta Crantz), apresentou biomassa das lagartas de 266,02 mg. Esse valor médio foi próximo

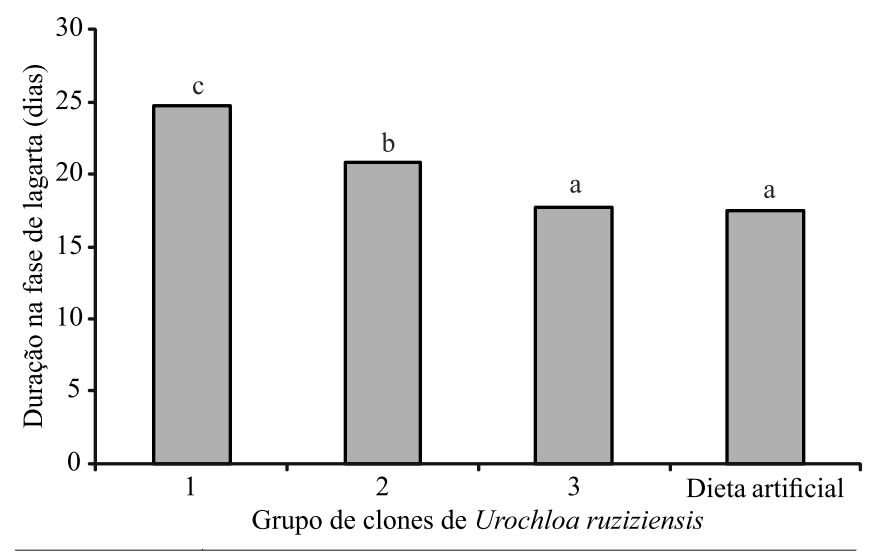

Grupo 1: $18,21,60,78,112$

Grupo 2: 3, 13, 25, 35, 49, 59, 70, 83, 85, 93, 116, 140 .

Grupo 3: 4, 6, 14, 15, 19, 28, 29, 34, 39, 42, 45, 54, 57, 61, 62, 66, 71, 73, 74, 76, $84,87,75,79,88,91,96,102,109,111,115,117,118,119,120,122,124$, $125,126,127,128,130,131,133,134,136,138,139,141,142,143,144$ $148,149$.

Figura 2. Duração média de lagartas de Spodoptera frugiperda alimentadas com diferentes clones de Urochloa ruziziensis. Médias seguidas de letras distintas diferem pelo teste de Scott-Knott, a 5\% de probabilidade, e formam os diferentes grupos de clones. aos de 25 clones do presente trabalho, nos quais o índice variou de 130 a $270 \mathrm{mg}$; para os demais clones, a variação foi de 270 a $420 \mathrm{mg}$.

Waquil et al. (2008) também constataram menor acúmulo de biomassa das pupas desenvolvidas em Brachiaria decumbens Stapf, do que em dieta artificial. Segundo Pencoe \& Martin (1981), o valor da biomassa de pupas é importante, uma vez que apresenta correlação direta com a fertilidade dos adultos. Dessa forma, em programas de melhoramento, pode-se inferir que o grupo 2 dos clones (Figura 3) deve ser preferido, em razão da possibilidade de redução da prole.

Ressalta-se que houve correlação significativa entre a duração e as biomassas das lagartas $(\mathrm{r}=-0,46$; $\mathrm{t}=-4,2423 ; \mathrm{p}=0,0001)$ e entre a biomassa das lagartas e a das pupas $(\mathrm{r}=0,43 ; \mathrm{t}=3,9353 ; \mathrm{p}=0,0002)$. Silveira et al. (1997) verificaram, em diferentes clones de milho, que a menor duração na fase de lagarta de $S$. frugiperda estava relacionada à maior biomassa; essa correlação também foi observada por Sá et al. (2009). Quanto mais curto for o período de desenvolvimento larval, sem redução da biomassa, mais adequado é o alimento ofertado para o desenvolvimento do inseto (Boregas et al., 2013). Os resultados obtidos no presente trabalho são indicativos de que, na sua maioria, os

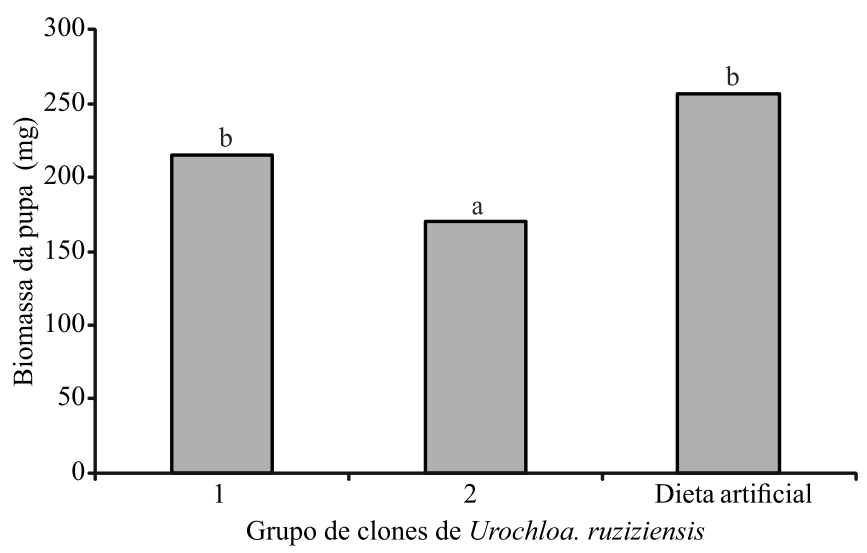

Grupo 1: $3,4,6,14,15,28,34,54,57,62,75,79,84,86,91,93,96,107,109,112$, $116,117,122,126,128,130,131,134,136,141,144,145,148,149$.

Grupo 2: 13, 18, 19, 25, 35, 39, 42, 49, 59, 60, 61, 66, 70, 71, 73, 74, 76, 78, $83,85,87,88,102,111,118,119,120,124,125,127,133,138,139$, $140,142,143$.

Figura 3. Biomassa das pupas advindas de lagartas de Spodoptera frugiperda alimentadas com diferentes clones de Urochloa ruziziensis. Médias seguidas de letras distintas diferem pelo teste de Scott-Knott, a 5\% de probabilidade, e formam os diferentes grupos de clones. 
clones de $U$. ruziziensis podem ser considerados alimentos pouco adequados ao desenvolvimento de lagartas de $S$. frugiperda. As lagartas não sobreviveram em diversos clones (Figura 1), e, mesmo nos que proporcionaram condições para o seu desenvolvimento, os insetos tiveram que aumentar o período larval como estratégia de sobrevivência (Figura 2); nessa condição, a biomassa das pupas também foi diminuída (Figura 3).

Além disso, os materiais dos grupos 1 e 2 proporcionaram maior duração de $S$. frugiperda e menor peso das lagartas, exceto os clones 49 e 21. Da mesma forma, aqueles que proporcionaram menor biomassa das lagartas também originaram menores pupas, exceto os clones 136, 112, 141, 96 e 75. Esses resultados confirmam as estimativas de correlação obtidas para as características envolvidas e mostraram o efeito prejudicial desses genótipos ao desenvolvimento do inseto, que apresentou maior período de desenvolvimento e menor biomassa. Assim, esses insetos estão mais suscetíveis a fatores bióticos e abióticos de mortalidade, o que é extremamente desejável no cenário de ILPF, em que uma cultura deve potencializar o desenvolvimento da outra.

O efeito conjunto dos parâmetros biológicos pôde ser observado com o índice de adaptação do inseto-praga (Figura 4). Baixos valores para o índice relativo de adaptação indicam que o alimento ofertado pelo hospedeiro é menos adequado para o desenvolvimento de $S$. frugiperda. Os valores desse índice variaram entre os clones avaliados, o que confirma as diferenças detectadas para as características sobrevivência e biomassa de lagartas e pupas (Figuras 1 a 4). Os clones de $U$. ruziziensis que apresentaram os menores índices de adaptação devem seguir no programa de melhoramento dessa espécie, para obtenção de novas cultivares que agreguem produtividade, qualidade da forragem e resistência à praga. Já os clones que apresentaram índices acima de 9,0, que correspondem a 33\% dos materiais avaliados, devem ser desconsiderados no programa de melhoramento, por potencializar a ocorrência de pragas no sistema de produção.

No Brasil, um dos fatores que pode estar contribuindo para a dificuldade do manejo de $S$. frugiperda é a grande oferta de hospedeiros ao longo do ano, seja pela sucessão de culturas (Barros et al., 2010) ou pelo emprego de cultivares suscetíveis ao inseto, o que caracteriza uma ponte verde, muito importante

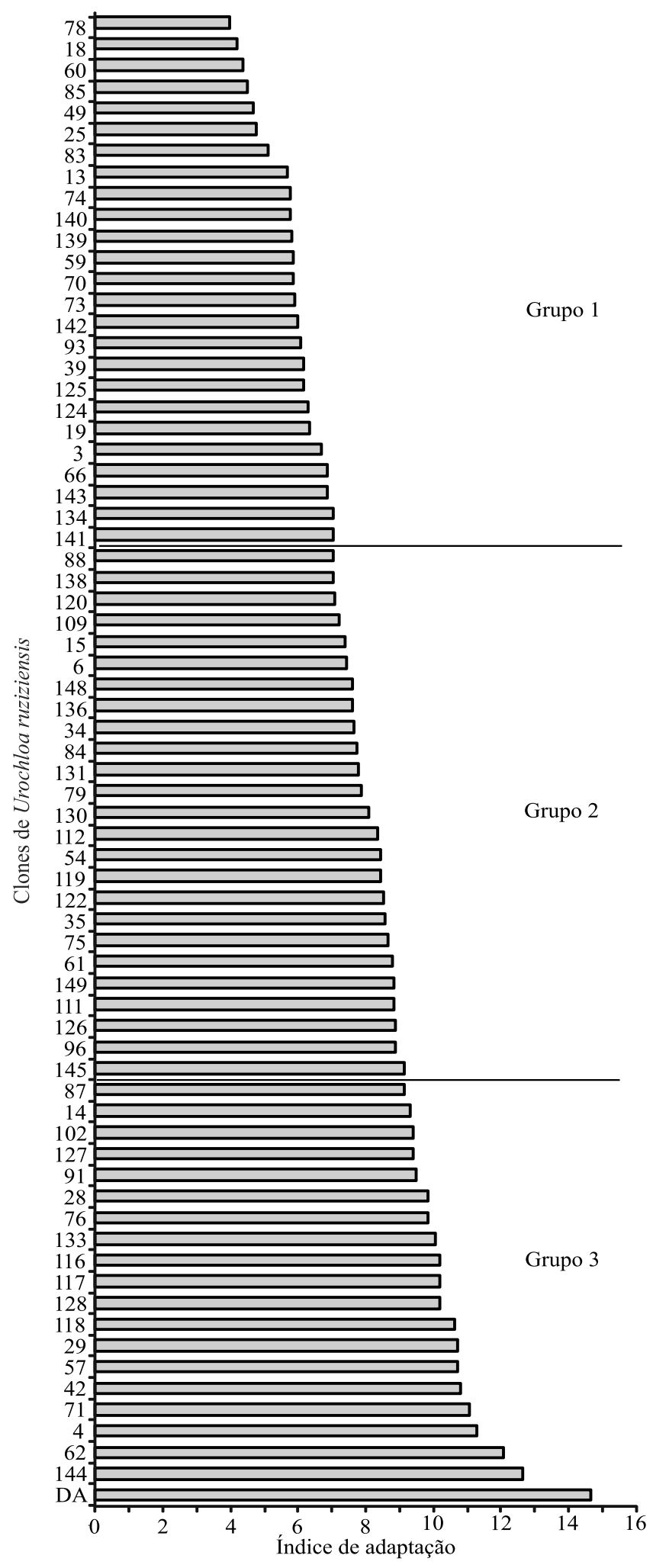

Figura 4. Índice de adaptação de lagartas de Spodoptera frugiperda alimentadas com diferentes clones de Urochloa ruziziensis. Os clones testados foram divididos em três grupos. DA, dieta artificial. 
para pragas polífagas. Este é o caso, por exemplo, do consórcio de lavouras de milho com forrageiras da espécie Urochloa, que, no presente trabalho, mostrou ser hospedeira relativamente favorável a $S$. frugiperda. Esse fato, associado ao comportamento alimentar polífago desse inseto-praga, facilita a sua multiplicação em diferentes espécies, durante a maior parte do ano (Sá et al., 2009; Campos et al., 2011).

Desse modo, enfatiza-se a importância da seleção de cultivares resistentes a $S$. frugiperda, quer sejam em milho quer em espécies forrageiras, para aumentar a sustentabilidade do sistema de produção. No caso do milho, recentemente tem aumentado o emprego de cultivares transgênicas contendo o alelo bt, que proporciona o controle do inseto. Já para as forrageiras, o conhecimento do grau de ataque das lagartas de $S$. frugiperda ainda é pequeno, pois esse inseto ainda não é considerado uma praga de importância para as forrageiras. Porém, tanto nos cultivos consorciados (ILPF) quanto nas áreas de pastagens, a tendência é que o ataque dos insetos e os danos causados sejam intensificados. Se a principal fonte de alimento dos insetos for retirada, pela substituição de cultivares suscetíveis de milho por outras resistentes, é natural que os insetos reduzam o potencial biótico ou migrem para outras culturas. Por isso, o conhecimento da resposta dos insetos à forrageira e à cultura consorciada é de fundamental importância para que o controle da praga seja realizado de modo eficiente. Meagher et al. (2004) sugeriram que os hospedeiros feijão-caupi [Vigna unguiculata (L.) Walp.] e crotalária (Crotalaria juncea L.) têm potencial para redução da população de $S$. frugiperda, ao proporcionar maior período de desenvolvimento e aumento da mortalidade larval.

No presente trabalho, foi possível evidenciar o potencial de utilização de alguns clones de $U$. ruziziensis para redução de populações de $S$. frugiperda. Os clones do grupo 3 (Figura 1), que apresentaram baixa sobrevivência das lagartas, e, entre os demais, os clones que proporcionaram ampliação do ciclo biológico, promoveram redução nas biomassas das pupas (Figuras 2, 3 e 4); portanto, deverão seguir no programa de melhoramento genético, com vistas à obtenção de cultivares resistentes às lagartas. A associação dessa característica em uma nova cultivar reforçará o potencial de utilização de $U$. ruziziensis no sistema de ILPF.

\section{Conclusão}

Há potencial de utilização de clones de Urochloa ruziziensis para redução de populações de Spodoptera frugiperda em sistema de integração lavoura-pecuária-floresta.

\section{Agradecimentos}

À Fundação de Amparo à Pesquisa do Estado de Minas Gerais (Fapemig) e ao Conselho Nacional de Desenvolvimento Científico e Tecnológico (CNPq), pelo apoio.

\section{Referências}

ALVARENGA,R.C.; PORFIRIO-DA-SILVA,V.; GONTIJONETO, M.M.; VILELA, L. Sistema integração lavoura-pecuária-floresta: condicionamento do solo e intensificação da produção de lavouras. Informe Agropecuário, v.31, p.59-67, 2010.

AYRES, M.; AYRES JUNIOR, M.; AYRES D.L.; SANTOS, A.S. BioEstat 3.0: aplicações estatísticas nas áreas das ciências biológicas e médicas. Belém: Sociedade Civil Mamirauá, 2003. 290p.

BALBINO, L.C.; CORDEIRO, L.A.M.; OLIVEIRA, P. de; KLUTHCOUSKI, J.; GALERANI, P.R.; VILELA, L. Agricultura sustentável por meio da integração lavoura-pecuária-floresta (ILPF). Informações Agronômicas, v.138, p.1-18, 2012.

BARROS, E.M.; TORRES, J.B.; BUENO, A.F. Oviposição, desenvolvimento e reprodução de Spodoptera frugiperda (J.E. Smith) (Lepidoptera: Noctuidae) em diferentes hospedeiros de importância econômica. Neotropical Entomology, v.39, p.996-1001, 2010. DOI: 10.1590/S1519-566X2010000600023.

BOREGAS, K.G.B.; MENDES, S.M.; WAQUIL, J.M.; FERNANDES, G.W. Estádio de adaptação de Spodoptera frugiperda (J. E. Smith) (Lepidoptera: Noctuidae) em hospedeiros alternativos. Bragantia, v.72, p.61-70, 2013. DOI: 10.1590/ S0006-87052013000100009.

BUSATO, G.R.; GRÜTZMACHER, A.D.; GARCIA, M.S.; GIOLO, F.P.; ZOTTI, M.J.; STEFANELLO JUNIOR, G.J. Biologia comparada de populações de Spodoptera frugiperda (J.E. Smith) (Lepidoptera: Noctuidae) em folhas de milho e arroz. Neotropical Entomology, v.34, p.743-750, 2005. DOI: 10.1590/ S1519-566X2005000500005.

CAMPOS, A.P. de; BOIÇA JUNIOR, A.L.; JESUS, F.G. de; GODOY, I.J. de. Avaliação de cultivares de amendoim para resistência a Spodoptera frugiperda. Bragantia, v.70, p.349-355, 2011. DOI: 10.1590/S0006-87052011000200014.

CASMUZ, A.; JUÁREZ, M.L.; SOCÍAS, M.G.; MURÚA, M.G.; PRIETO, S.; MEDINA, S.; WILLINK, E.; GASTAMINZA, G. Revisión de los hospederos del gusano cogollero del maíz, Spodoptera frugiperda (Lepidoptera: Noctuidae). Revista da la Sociedad Entomológica Argentina, v.69, p.209-231, 2010. 
FIGUEIREDO, M. de L.C.; MARTINS-DIAS, A.M.P.; CRUZ, I. Relação entre a lagarta-do-cartucho e seus agentes de controle biológico natural na produção de milho. Pesquisa Agropecuária Brasileira, v.41, p.1693-1698, 2006. DOI: 10.1590/ S0100-204X2006001200002.

GONTIJO NETO, M.M.; VIANA, M.C.M.; ALVARENGA, R.C.; SANTOS, E.A. dos; SIMÃO, E. de P.; CAMPANHA, M.M. Sistema de integração lavoura-pecuária-floresta em Minas Gerais. Boletim de Indústria Animal, v.71, p.183-191, 2014. DOI: 10.17523/bia.v71n2p183.

LOPES, G. da S.; LEMOS, R.N.S. de; MACHADO, K.K.G.; MACIEL, A.A.S.; OTTATI, A.L.T. Biologia de Spodoptera frugiperda (J. Smith) (Lepidoptera: Noctuidae) em folhas de mandioca (Manihot esculenta, Crantz). Revista Caatinga, v.21, p.134-140, 2008.

MEAGHER, R.L.; NAGOSHI, R.N.; STUHL, C.; MITCHELL, E.R. Larval development of fall armyworm (Lepidoptera: Noctuidae) on different cover crop plants. Florida Entomologist, v.87, p.454-460, 2004. DOI: 10.1653/0015-4040(2004)087[0454:LDO FAL]2.0.CO;2.

PASHLEY, D.P.; HARDY, T.N.; HAMMOND, A.M. Host effects on developmental and reproductive traits in fall armyworm strains (Lepidoptera: Noctuidae). Annals of the Entomological Society of America, v.88, p.748-755, 1995. DOI: 10.1093/ aesa/88.6.748.
PENCOE, N.L.; MARTIN, P.B. Development and reproduction of fall armyworms on several wild grasses. Environmental Entomology, v.10, p.999-1002, 1981. DOI: 10.1093/ee/10.6.999.

SÁ, V.G.M. de; FONSECA, B.V.C.; BOREGAS, K.G.B.; WAQUIL, J.M. Sobrevivência e desenvolvimento larval de Spodoptera frugiperda (J E Smith) (Lepidoptera: Noctuidae) em hospedeiros alternativos. Neotropical Entomology, v.38, p.108-115, 2009. DOI: 10.1590/S1519-566X2009000100012.

SILVEIRA, L.C.P.; VENDRAMIM, J.D.; ROSSETTO, C.J. Efeito de genótipos de milho no desenvolvimento de Spodoptera frugiperda (J.E. Smith). Anais da Sociedade Entomológica do Brasil, v.26, p.291-298, 1997. DOI: 10.1590/S0301-80591997000200010.

SOUZA SOBRINHO, F. de; AUAD, A.M. Genetic improvement of Brachiaria ruziziensis at Embrapa Dairy Cattle. In: JANK, L.; CHIARI, L.; VALLE, C.B. do; SIMEÃO, R.M. (Eds.). Forage breeding and biotechnology. Brasília: Embrapa, 2013, p.59-75.

WAQUIL, J.M.; BORGAS, K.G.B.; MENDES, S.M. Viabilidade do uso de hospedeiros alternativos como área de refúgio para o manejo da resistência da lagarta-do-cartucho, Spodoptera frugiperda (J. E. Smith) (Lepidoptera: Noctuidae) no cultivo do milho Bt. Sete Lagoas: Embrapa Milho e Sorgo, 2008. 10p. (Embrapa Milho e Sorgo. Comunicado técnico, 160).

WAQUIL, J.M.; VILELA, F.M.F.; FOSTER, J.E. Resistência do milho (Zea mays L.) transgênico (Bt) à lagarta-do-cartucho, Spodoptera frugiperda (Smith) (Lepidoptera: Noctuidae). Revista Brasileira de Milho e Sorgo, v.1, p.1-11, 2002. DOI: 10.18512/1980-6477/rbms.v1n3p1-11.

$\overline{\text { Recebido em } 27 \text { de novembro de } 2014 \text { e aprovado em } 11 \text { de fevereiro de } 2016}$ 